\title{
Analysis of Social Determinants and the Utilization of Pediatric Tele-Urgent Care During the COVID-19 Pandemic: Cross-sectional Study
}

Saif Khairat ${ }^{1,2,3}$, MPH, PhD; Phillip McDaniel ${ }^{4}$, MA; Matthew Jansen ${ }^{4}$, MS; Tia Francis ${ }^{4}$, MS; Barbara Edson ${ }^{5}$, RN, MBA; Robert Gianforcaro ${ }^{5}$, DO

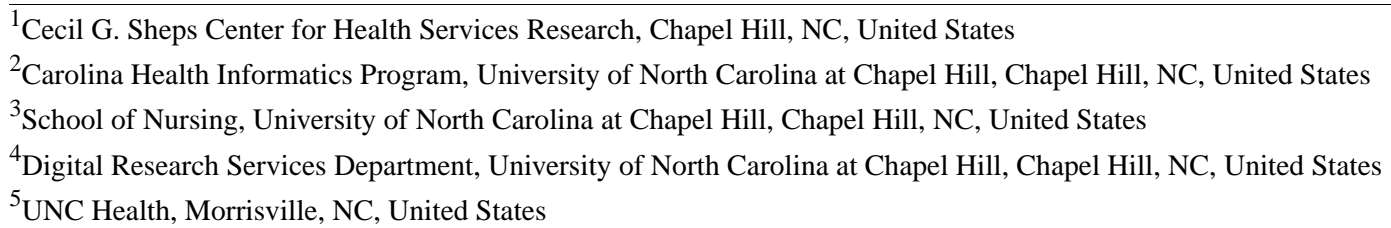

\section{Corresponding Author:}

Saif Khairat, MPH, PhD

Cecil G. Sheps Center for Health Services Research

725 M.L.K. Jr Blvd

Chapel Hill, NC, 27516

United States

Phone: 19198435413

Email: saif@unc.edu

\begin{abstract}
Background: Telehealth is increasingly used to provide specialty consultations to infants and children receiving care. However, there is uncertainty if the COVID-19 pandemic has influenced the use of telehealth among vulnerable populations.

Objective: This research aims to compare the overall use of tele-urgent care visits for pediatric patients before and after the pandemic, especially among vulnerable populations.

Methods: We conducted a cross-sectional analysis of pediatric tele-urgent care visits at a virtual care center at a southeastern health care center. The main outcome of this study was the use of pediatrics tele-urgent visits across geographical regions with different levels of social disparities and between 2019 and 2020.

Results: Of 584 tele-urgent care visits, 388 (66.4\%) visits occurred in 2020 during the pandemic compared to $196(33.6 \%)$ visits in 2019. Among 808 North Carolina zip codes, 181 (22\%) consisted of a high concentration of vulnerable populations, where $17.7 \%$ (56/317) of the tele-urgent care visits originated from. The majority $(215 / 317,67.8 \%)$ of tele-urgent care visits originated from zip codes with a low concentration of vulnerable populations. There was a significant association between the rate of COVID-19 cases and the concentration level of social factors in a given Zip Code Tabulation Area.

Conclusions: The use of tele-urgent care visits for pediatric care doubled during the COVID-19 pandemic. The majority of the tele-urgent care visits after COVID-19 originated from regions where there is a low presence of vulnerable populations. In addition, our geospatial analysis found that geographic regions with a high concentration of vulnerable populations had a significantly higher rate of COVID-19-confirmed cases and deaths compared to regions with a low concentration of vulnerable populations.
\end{abstract}

(JMIR Pediatr Parent 2021;4(3):e25873) doi: 10.2196/25873

\section{KEYWORDS}

telehealth; pediatrics; social; determinants; COVID-19; use; children; infant; consultation; telemedicine; urgent care; vulnerable population; cross-sectional; minority 


\section{Introduction}

Pediatricians have used telehealth to provide a broad range of health care services among primary and specialty care [1-3]. Telehealth is increasingly used to provide specialty consultations to infants and children receiving care [4]. However, since the pandemic, the use of tele-urgent care for pediatrics has grown to include telephone consultations and remote surveillance or to replace in-person primary care visits without sufficient evaluation of these interventions [5-7]. There is limited knowledge on the use of tele-urgent care to respond to urgent care needs in pediatrics during the COVID-19 pandemic.

Social determinants of health are paramount to the use of tele-urgent care because of the widening digital divide that occurs as a result of differences in individual socioeconomic characteristics [8]. Social determinants of health can be defined as the circumstances that impact the health of individuals from birth to death including socioeconomic, educational, and access to health care [9]. Prior to the COVID-19 pandemic, health disparities and inequity demonstrated differences in adoption of tele-urgent care among vulnerable populations [10,11]. Vulnerable populations are defined as populations that are at risk for health access because of economic, ethnic, or health characteristics [12]. In this study, we refer to vulnerable populations based on race, socioeconomic status, and health insurance status. There is uncertainty if the COVID-19 pandemic has influenced the use of tele-urgent care among vulnerable populations. Therefore, the objective of this study was to compare the overall use of tele-urgent care services for pediatric patients before and after the COVID-19 pandemic especially among vulnerable populations.

\section{Methods}

\section{Overview}

We conducted a cross-sectional analysis of pediatric tele-urgent care visits at a virtual care center at a southeastern health care center. The virtual care center offers on-demand services to all patients older than 2 years regardless of their geographic location or medical affiliation to a health care system. Patients are required to create a profile through the virtual care center web portal and provide personal information such as age, gender, residential address, and insurance coverage. The web portal allows patients to choose from a list of providers based on the patient's chief complaint. In addition, patients can choose between having an on-demand visit or scheduling a visit based on their preference and the availability of the provider. Board-certified physicians are available for on-demand televisits with the ability to prescribe and send medications to the patient's choice of pharmacy.

\section{Data and Materials}

The tele-urgent care visit data were received and preprocessed in Excel (Microsoft Corporation). All patients between the ages of 2 and 18 years were included in the analysis. Gender had three categories that were male, female, and nonbinary. Insurance type included the member ID and group ID if the patient provided insurance coverage information. Otherwise, the insurance field was empty, indicating the patient reported no insurance coverage.

\section{Outcomes}

The main outcome of this study was the use of pediatrics tele-urgent care visits across geographical regions with different levels of social disparities and between 2019 and 2020.

\section{Data Analysis}

Since tele-urgent care participant data were collected and available at the zip level, Zip Code Tabulation Areas (ZCTAs; generalized areal representations of United States Postal Service zip code service areas) were used as the unit of analysis [13]. A variety of detailed demographic data are available at the ZCTA level from the American Community Survey (ACS) [14]. We developed social factors based on previous social determinants of health models [15], including our own model to assess health disparities in the use of tele-urgent care [10]. We collected daily COVID-19 case counts in North Carolina zip codes during the study period to assess if there was a relationship between the prevalence of COVID-19 and the use of tele-urgent care for pediatrics within North Carolina zip codes.

For this research, ZCTA-level social factors data was obtained from the ACS 2014-2018 5-year estimates, the most current 5-year data available from the ACS. We used percentages to account for population density for each social factor. Social factors used in the analyses were percent American Indian or Alaska Native people, percent of Black or African American people, percent in poverty, percent of single female headed households receiving Supplemental Nutrition Assistance Program (SNAP; formerly known as food stamps) with children younger than 18 years, percent of households receiving food stamps or SNAP with a person older than 60 years, total population receiving Medicare, and total population receiving Medicaid. For each variable, a threshold was set to determine if a ZCTA was at risk (ZCTAs with a value over the threshold were coded with a 1 , while those below were coded with a 0 ). Scores across all factors were tabulated for all ZCTAs to create an aggregate risk and deprivation score (higher aggregate scores indicate greater risk and deprivation). The coding of ZCTA counts were not mutually exclusive among social factors.

The maps were created using the computed social score for each ZCTA, as well as data on 2019 and 2020 telemedicine visits, and COVID-19 cases, aggregated to ZCTAs. For reference, urban centers throughout the state are labeled on the 2019 visits map. We used dot plot graphs to represent the number of visits coming from zip codes tagged with each of the listed social factors, tagged with no social factors, and the overall visit counts for each year for reference. Factors were sorted by the overall frequency of visits across both years.

Descriptive statistics, visualizations, and statistical tests were all performed in $\mathrm{R}$ ( $\mathrm{R}$ Foundation for Statistical Computing) using ggplot2, version 4.0.2., and the maps were created using ArcGIS Pro 2.6.0 (Esri; July 28, 2020) and Illustrator 2020 24.3.0 (Adobe Inc; August 1, 2020). We primarily analyzed the data through descriptive tables and visualizations. 


\section{Results}

Of 584 tele-urgent care visits, $388(66.4 \%)$ visits occurred in 2020 during the pandemic compared to 196 (33.6\%) visits in 2019. Over half of the patients were male $(112 / 196,57 \%)$ in
2019 , versus in 2020 when over half of the patients were female $(202 / 388,52 \%)$. For both years, the majority of patients reported having health insurance coverage. There was a larger gap between insured and uninsured patients in 2020, such that there were $265(68 \%)$ insured patients and $123(32 \%)$ uninsured patients (Table 1).

Table 1. Patient characteristics of tele-urgent care visits between March 1 and September 30, 2019 and 2020.

\begin{tabular}{lllll}
\hline Variables & 2019 visits $(\mathrm{n}=196), \mathrm{n}(\%)$ & 2020 visits $(\mathrm{n}=388), \mathrm{n}(\%)$ & Ratio of increase & Total $(\mathrm{N}=584), \mathrm{n}(\%)$ \\
\hline $\begin{array}{l}\text { Gender } \\
\quad 112(57.1)\end{array}$ & \multicolumn{1}{l}{$\begin{array}{l}\text { Male } \\
\text { Female }\end{array}$} & $183(47.2)$ & 1.63 & $295(50.5)$ \\
$\quad$ Nonbinary & $0(0.0)$ & $202(52.1)$ & 2.4 & $3(0.5)$ \\
Insurance coverage & & $3(0.8)$ & 3 & $373(63.9)$ \\
$\quad$ Insured & $108(55.1)$ & $265(68.3)$ & $2.45)$ \\
$\quad$ Uninsured & $88(44.9)$ & $123(31.7)$ & 1.39 & $211(36.1)$ \\
\hline
\end{tabular}

The line graph in Figure 1 shows the tele-urgent care use over time broken up by social flag categories. Most of the groups show a clear increase in visits in 2020 compared to 2019. The pattern of visits over time (a peak in March followed by variable but generally lower visit counts in April through August) were not remarkably different between the time periods.

This line graph shows the percentage of the total visits across the March to August time frame within the overall telemedicine population and broken down into the zip codes with and without social factors identified. The year 2019 shows more variation around the overall trend, reflecting a year not driven by COVID-19 and the smaller overall number of visits.

Visits from 2019 and 2020 were also compared geographically. Figure 2 compares the number of tele-urgent care visits in each year against the social deprivation score for each zip code. Overall, there was an increase in visits in 2020, with much of the increase occurring in the central part of the state (Raleigh-Durham-Cary), an area with high population density with large medical centers. The areas colored in light gray demonstrate the ZCTAs with low social factors, while the areas colored in dark green represent areas with high social factors.

The number of tele-urgent care visits doubled in $2020(n=317)$ compared to $2019(n=155)$. In addition, the distribution of the visits covered more geographic locations in North Carolina compared to the visits in 2019 that were primarily around the Durham-Raleigh area (Figure 3A and B). The northern and southern part of the North Carolina include zip codes with higher social flag scores, which indicates areas of higher social vulnerability (Figure 3C). The same regions with a high social flag score also experienced high rates of COVID-19-confirmed cases, which presents a challenge to an already vulnerable population (Figure 3D).

Figure 1. Line graph presenting pediatrics tele-urgent care visits falling under each social flag during 2019 and 2020.

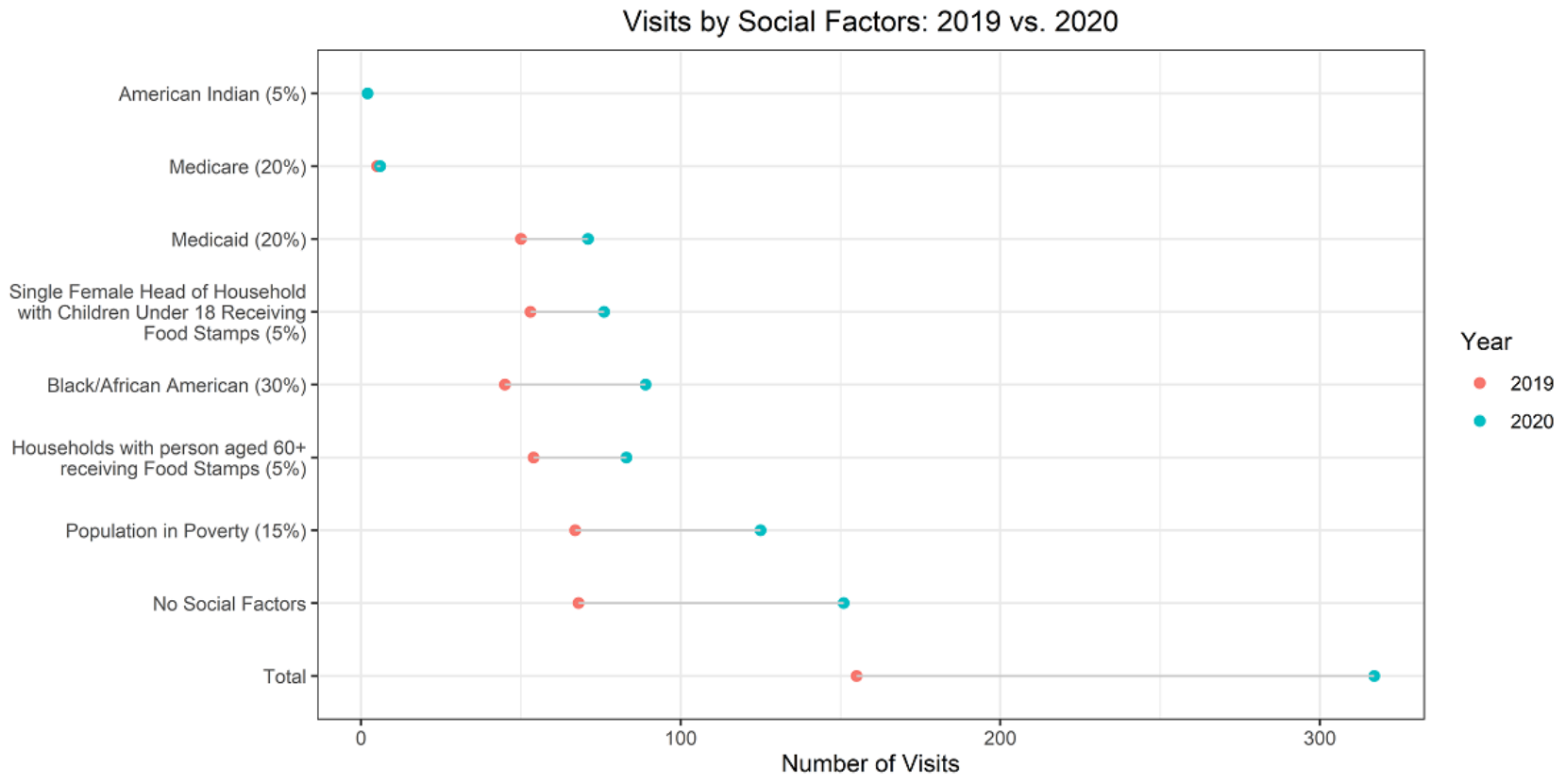


Figure 2. Bivariate choropleth map comparing the number of visits per 10,000 residents younger than 17 years against social flag scores. Figure produced using ArcGIS Pro 2.6.0 (July 28, 2020) and Illustrator 2020 24.3.0 (August 1, 2020).

\section{Visits}
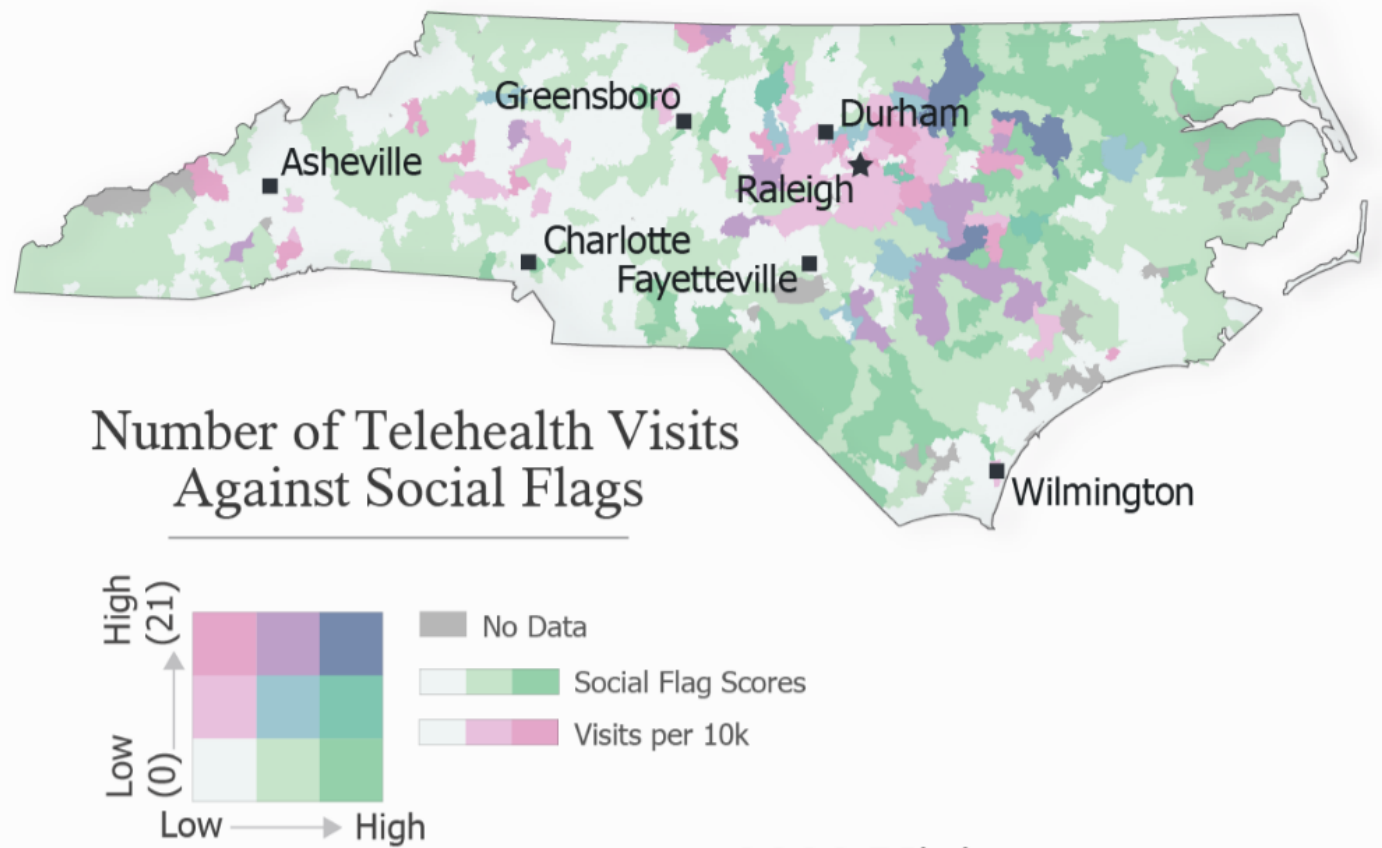

(0) (6)

\section{Visits}

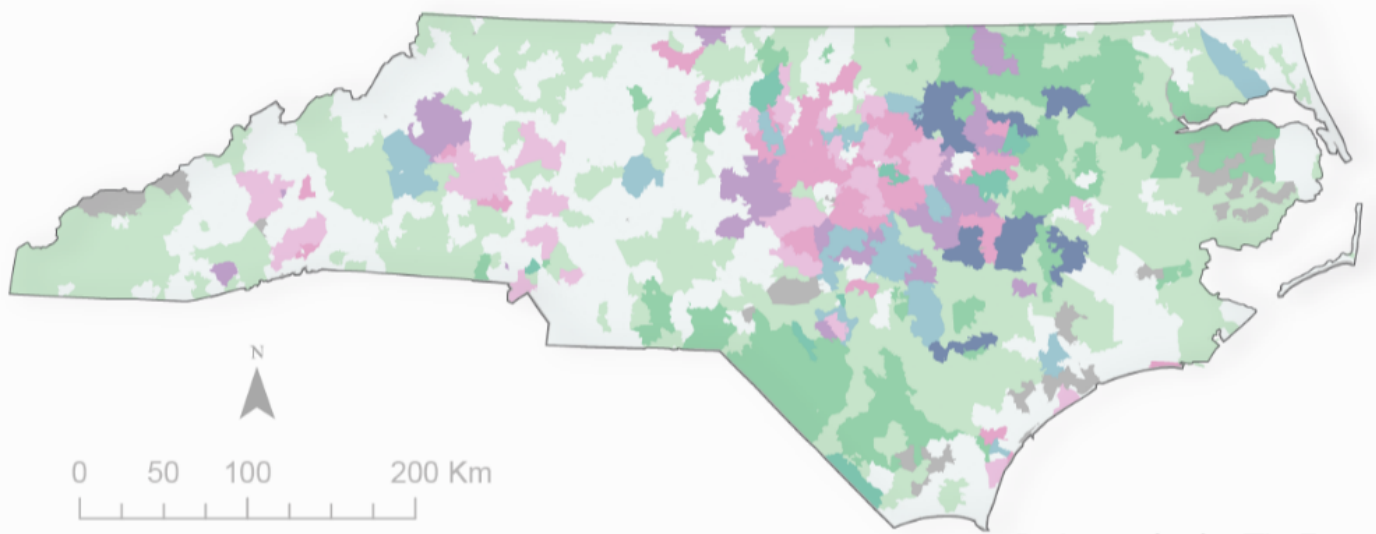


Figure 3. (A) Map of the tele-urgent care pediatric visits in 2019, (B) the tele-urgent care pediatric visits in 2020, (C) the categorization of North Carolina zip codes based on social vulnerability factors, (D) map of North Carolina with COVID-19-confirmed cases in 2020 by zip code.

A.

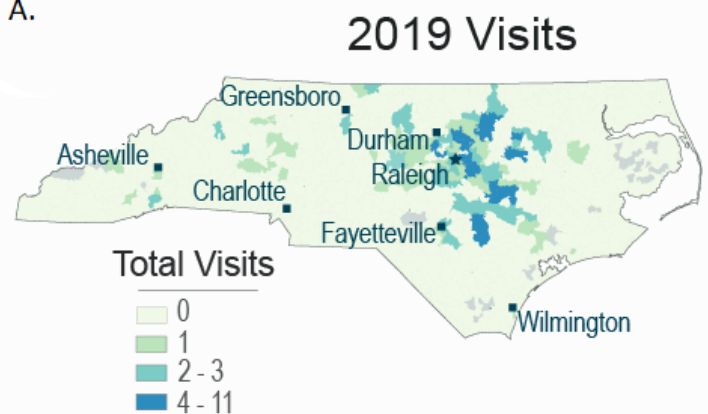

C.

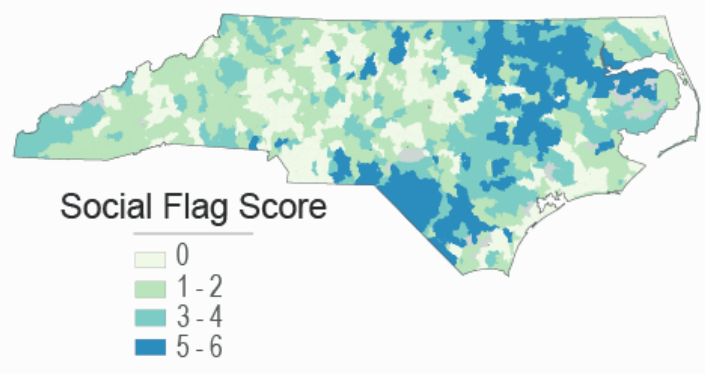

B.
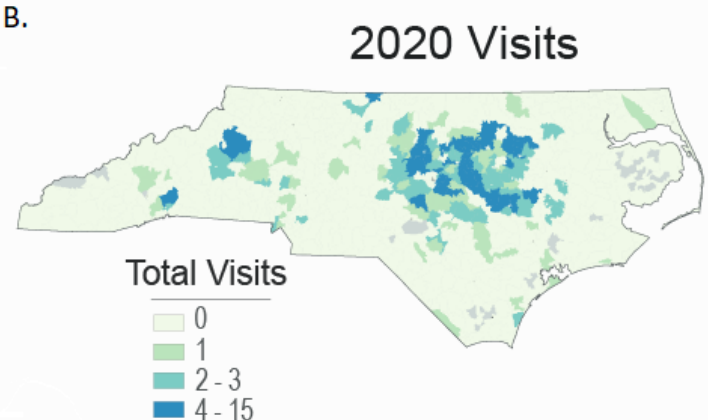

D.

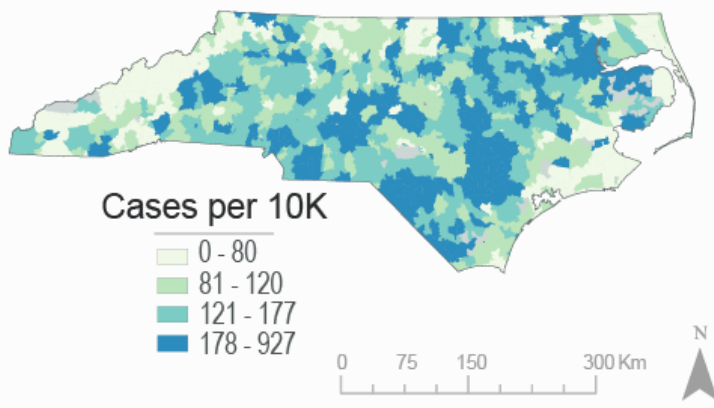

Among 808 North Carolina zip codes, 181 (22\%) consisted of a high concentration of vulnerable populations, where $17.7 \%$ $(56 / 317)$ of the tele-urgent care visits originated from. The majority $(215 / 317,67.8 \%)$ of tele-urgent care visits originated from zip codes with a low concentration of vulnerable populations. Areas of high concentration of vulnerable populations experienced the highest rates of COVID-19 cases (214.8) and deaths (4.1) per 10,000. association between the rate of COVID-19 cases and the concentration level of social factors in a given ZCTA.

Table 2. The use of tele-urgent care services among low, medium, and high regions of social vulnerability between March 1 and September 30, 2019 and 2020.

\begin{tabular}{llllllll}
\hline $\begin{array}{l}\text { Social flag } \\
\text { score }\end{array}$ & $\begin{array}{l}\text { Zip } \\
\text { codes, } \mathrm{n}\end{array}$ & $\begin{array}{l}\text { Telehealth visits } \\
\text { Mar-Aug 2019, } \mathrm{n} \\
(\%)\end{array}$ & $\begin{array}{l}\text { Telehealth visits } \\
\text { per 10,000 Mar- } \\
\text { Aug 2019, } \mathrm{n}\end{array}$ & $\begin{array}{l}\text { Telehealth visits } \\
\text { Mar-Aug 2020, } \mathrm{n} \\
(\%)\end{array}$ & $\begin{array}{l}\text { Telehealth visits } \\
\text { per 10,000 Mar- } \\
\text { Aug 2020, } \mathrm{n}\end{array}$ & $\begin{array}{l}\text { COVID-19-con- } \\
\text { firmed cases per } \\
10,000, \mathrm{n}\end{array}$ & $\begin{array}{l}\text { COVID-19 } \\
\text { deaths per } \\
10,000, \mathrm{n}\end{array}$ \\
\hline Low (0-1) & 417 & $93(60)$ & 0.71 & $215(67.8)$ & 1.64 & 138.2 & 2.0 \\
Medium (2-3) & 210 & $19(12.3)$ & 0.4 & $46(14.5)$ & 0.98 & 175.6 & 2.8 \\
High (4-7) & 181 & $43(27.7)$ & 0.9 & $56(17.7)$ & 1.18 & 1.9 & 4.1 \\
Total & 808 & 155 & 0.69 & 317 & 1.4 & 161.4 & 2.6 \\
\hline
\end{tabular}

\section{Discussion}

\section{Principal Findings}

We conducted a cross-sectional study of pediatrics tele-urgent care visits before and after the COVID-19 pandemic among vulnerable populations. We found that the volume of tele-urgent care visits for pediatrics doubled after the pandemic when compared to the year before, which can be explained by the shutdown of health care systems during the initial phases of the COVID-19 pandemic. Post-COVID-19, there was a substantial shift in patient characteristics seeking tele-urgent care for pediatrics such that there were more women and patients with health insurance coverage compared to pre-COVID-19. Most of the postpandemic visits originated from the metropolitan Raleigh region. This could be explained by several reasons including the shutdown of in-person nonessential visits during the initial phases of the pandemic. In addition, there were strict COVID-19 social gathering restrictions, and many schools transitioned to virtual classrooms.

Post-COVID-19, we reported an overall substantial increase in pediatric tele-urgent care visits. Regions characterized with high poverty and high concentration of African American people encountered the highest increase in visits among all examined social factors. The high demand among these communities 
demonstrates high disparities for tele-urgent care use for pediatrics care during the pandemic. We recommend future exploration of current infrastructure and culture to adopting tele-urgent care such that patients living in areas of high poverty may not have access to tele-urgent care equipment or internet access, which may hinder the adoption of tele-urgent care among vulnerable populations. Therefore, offering video and telephone visit options to vulnerable populations may improve adoption and use levels of tele-urgent care.

Telehealth provides a convenient delivery method of health care for pediatric patients; however, the impact of tele-urgent care on patient outcomes remains unknown. We recommend future investigation of the effect of tele-urgent care on emergency department visits and urgent care clinic visits. Moreover, prescription rates were found to be different between video and telephone visits [16]. We recommend more investigations around medication prescription rates in tele-urgent care visits compared to in-person visits for pediatric care.

There appears to be a relationship between social flags and change in tele-urgent care visits between 2019 and 2020. Although a sizeable minority of tele-urgent care visits occurred in regions with high concentrations of vulnerable populations, the majority of tele-urgent care visits occurred in zip codes with low concentrations of vulnerable populations. Post-COVID-19, the volume of tele-urgent care visits in regions with a high concentration of vulnerable populations was less when compared to pre-COVID-19. Although the reason for such a drop in visits is unclear, it is possible that loss of employment and new local tele-urgent care clinics may have attributed to the decrease in visits. In addition, it is possible that during the pandemic newly established tele-urgent care services available through local clinics and primary care providers were preferred by patients.

Post-COVID-19, there were two spikes in the volume of tele-urgent care visits in March and July 2020 among most vulnerable population groups compared to two spikes in 2019 in March and April. The post-COVID-19 increase may be associated with the sudden shutdown of in-person appointments across the state in March 2020 in response to the World Health Organization announcing COVID-19 as a global pandemic [17]. Another explanation to the spikes in the month of March could be associated with the influenza and allergy seasons, which may explain the spike in both 2019 and 2020. In addition, the end of the school year coupled with the national holiday the Fourth of July may have attributed to the increase in visit volume during the month of July. Telehealth can be a suitable intervention to manage chronic care conditions within pediatric patients who may lack access during a pandemic due to the shutdown of schools and clinics [18].

Telehealth use in pediatric care has shown major increase in use during the pandemic. In the future, telehealth may be a suitable health care delivery modality that complements in-office pediatric visits for established patients. Although telehealth use has increased during the pandemic, there remains unanswered questions around the effectiveness of telehealth in pediatric care and the quality of care [19]. We recommend the integration of geospatial technologies to evaluate access factors such as broadband access, clinical effectiveness, medication prescription rates, and the acceptance of telehealth among pediatric patients and providers.

\section{Limitations}

This study has several limitations. Although the tele-urgent care data is statewide, it represents a single virtual care center. The patient demographic form did not include ethnic or racial information, which limited our ability to map patient-level ethnic data to zip code-level ethnic data. In the future, patient demographic forms will include ethnic and racial fields. The comparison of 2019 and 2020 data may include confounding factors including the effect of marketing campaigns and word of mouth in increasing the volume of tele-urgent care visits.

\section{Conclusion}

The use of tele-urgent care visits for pediatric care doubled during the COVID-19 pandemic. The majority of the tele-urgent care visits after COVID-19 originated from regions where there is a low presence of vulnerable populations. In addition, our geospatial analysis found that geographic regions with a high concentration of vulnerable populations had a significantly higher rate of COVID-19-confirmed cases and deaths compared to regions with a low concentration of vulnerable populations.

\section{Conflicts of Interest}

None declared.

\section{References}

1. Hakim A, Gaviria-Agudelo C, Edwards K, Olson D, PIDS Telehealth Working Group. Pre-coronavirus disease 2019 telehealth practices among pediatric infectious diseases specialists in the United States. J Pediatric Infect Dis Soc 2021 Apr 30;10(4):485-491 [FREE Full text] [doi: 10.1093/jpids/piaa146] [Medline: 33196843]

2. Gali K, Joshi S, Hueneke S, Katzenbach A, Radecki L, Calabrese T, et al. Barriers, access and management of paediatric epilepsy with telehealth. J Telemed Telecare 2020 Nov 12:1357633X20969531 [FREE Full text] [doi: 10.1177/1357633X20969531] [Medline: 33183129]

3. Allen HI, Gillespie P, Vazquez-Ortiz M, Murphy AW, Moylett EM. A cost-analysis of outpatient paediatric penicillin allergy de-labelling using telemedicine. Clin Exp Allergy 2021 Mar;51(3):495-498. [doi: 10.1111/cea.13782] [Medline: 33170984]

4. Burke BL, Hall RW, Section on Telehealth Care. Telemedicine: pediatric applications. Pediatrics 2015 Jul;136(1):e293-e308 [FREE Full text] [doi: 10.1542/peds.2015-1517] [Medline: 26122813] 
5. Sharawat IK, Panda PK. Caregiver satisfaction and effectiveness of teleconsultation in children and adolescents with migraine during the ongoing COVID-19 pandemic. J Child Neurol 2021 Mar;36(4):296-303. [doi: 10.1177/0883073820968653] [Medline: 33170754 ]

6. Ozsezen B, Emiralioglu N, Tural D, Sunman B, Buyuksahin H, Yalcin E, et al. Telephone surveillance during 2019 novel coronavirus disease: is it a helpful diagnostic tool for detecting acute pulmonary exacerbations in children with chronic lung disease? J Telemed Telecare 2020 Nov 12:1357633X20972008. [doi: 10.1177/1357633X20972008] [Medline: 33183128]

7. Macy ML, Huetteman P, Kan K. Changes in primary care visits in the 24 weeks after COVID-19 stay-at-home orders relative to the comparable time period in 2019 in metropolitan Chicago and northern Illinois. J Prim Care Community Health 2020;11:2150132720969557 [FREE Full text] [doi: 10.1177/2150132720969557] [Medline: $\underline{33174495]}$

8. Marcin JP, Shaikh U, Steinhorn RH. Addressing health disparities in rural communities using telehealth. Pediatr Res 2016 Jan;79(1-2):169-176. [doi: 10.1038/pr.2015.192] [Medline: 26466080]

9. Braveman P, Gottlieb L. The social determinants of health: it's time to consider the causes of the causes. Public Health Rep 2014;129 Suppl 2:19-31 [FREE Full text] [doi: 10.1177/00333549141291S206] [Medline: 24385661]

10. Khairat S, Haithcoat T, Liu S, Zaman T, Edson B, Gianforcaro R, et al. Advancing health equity and access using telemedicine: a geospatial assessment. J Am Med Inform Assoc 2019 Aug 01;26(8-9):796-805 [FREE Full text] [doi: 10.1093/jamia/ocz108] [Medline: $\underline{31340022}$ ]

11. Hamad J, Fox A, Kammire MS, Hollis AN, Khairat S. Evaluating the experiences of new and existing teledermatology patients during the COVID-19 pandemic: cross-sectional survey study. JMIR Dermatol 2021;4(1):e25999 [FREE Full text] [doi: 10.2196/25999] [Medline: 34028471$]$

12. Waisel D. Vulnerable populations in healthcare. Curr Opin Anaesthesiol 2013 Apr;26(2):186-192. [doi: 10.1097/ACO.0b013e32835e8c17] [Medline: 23385323]

13. ZIP Code Tabulation Areas (ZCTAs). United States Census Bureau. 2020. URL: https://www.census.gov/programs-surveys/ geography/guidance/geo-areas/zctas.html [accessed 2020-11-28]

14. About the American Community Survey. United States Census Bureau. 2020. URL: https://www.census.gov/ programs-surveys/acs/about.html [accessed 2020-08-18]

15. Kolak M, Bhatt J, Park YH, Padrón NA, Molefe A. Quantification of neighborhood-level social determinants of health in the continental United States. JAMA Netw Open 2020 Jan 03;3(1):e1919928 [FREE Full text] [doi:

10.1001/jamanetworkopen.2019.19928] [Medline: 31995211]

16. Khairat S, Liu S, Zaman T, Edson B, Gianforcaro R. Factors determining patients' choice between mobile health and telemedicine: predictive analytics assessment. JMIR Mhealth Uhealth 2019 Jun 08;7(6):e13772 [FREE Full text] [doi: 10.2196/13772] [Medline: 31199332]

17. Khairat S, Meng C, Xu Y, Edson B, Gianforcaro R. Interpreting COVID-19 and virtual care trends: cohort study. JMIR Public Health Surveill 2020 Apr 15;6(2):e18811 [FREE Full text] [doi: 10.2196/18811] [Medline: 32252023]

18. Serlachius A, Badawy SM, Thabrew H. Psychosocial challenges and opportunities for youth with chronic health conditions during the COVID-19 pandemic. JMIR Pediatr Parent 2020 Oct 12;3(2):e23057 [FREE Full text] [doi: 10.2196/23057] [Medline: 33001834]

19. Badawy SM, Radovic A. Digital approaches to remote pediatric health care delivery during the COVID-19 pandemic: existing evidence and a call for further research. JMIR Pediatr Parent 2020 Jun 25;3(1):e20049 [FREE Full text] [doi: 10.2196/20049] [Medline: 32540841]
Abbreviations
ACS: American Community Survey
SNAP: Supplemental Nutrition Assistance Program
ZCTA: Zip Code Tabulation Area

Edited by $S$ Badawy, MD, MS; submitted 16.12.20; peer-reviewed by J op den Buijs, C Rea; comments to author 11.04 .21 ; revised
version received 27.05.21; accepted 21.06.21; published 30.08.21
Please cite as:
Khairat $S$, McDaniel P, Jansen M, Francis T, Edson B, Gianforcaro $R$
Analysis of Social Determinants and the Utilization of Pediatric Tele-Urgent Care During the COVID-19 Pandemic: Cross-sectional
Study
JMIR Pediatr Parent 2021;4(3):e25873
URL: $\underline{\text { https://pediatrics.jmir.org/2021/3/e25873 }}$
doi: $10.2196 / 25873$
PMID:


CSaif Khairat, Phillip McDaniel, Matthew Jansen, Tia Francis, Barbara Edson, Robert Gianforcaro. Originally published in JMIR Pediatrics and Parenting (https://pediatrics.jmir.org), 30.08.2021. This is an open-access article distributed under the terms of the Creative Commons Attribution License (https://creativecommons.org/licenses/by/4.0/), which permits unrestricted use, distribution, and reproduction in any medium, provided the original work, first published in JMIR Pediatrics and Parenting, is properly cited. The complete bibliographic information, a link to the original publication on https://pediatrics.jmir.org, as well as this copyright and license information must be included. 